\title{
Fibroma ossificante periférico: relato de caso
}

\author{
Peripheral ossifying fibroma: case report
}

Fibroma osificante periférico. reporte de un caso

\author{
Lucieni Cristina Trovati MORETI ${ }^{\mathbf{1}}$ \\ Shirley Tatiane Martins PIMENTEL ${ }^{2}$ \\ Nilton César Pezati BOER ${ }^{\mathbf{1}}$ \\ Rita de Cássia VILARIM ${ }^{3}$ \\ Karina Gonzales Câmara FERNANDES ${ }^{\mathbf{1}}$ \\ ${ }^{1}$ Professor(a) do Departamento de Endodontia \\ Faculdade de Odontologia, Universidade Camilo Castelo Branco (UNICASTELO), Campus Fernandópolis- SP Brasil \\ ${ }^{2}$ Graduada em Odontologia, \\ Faculdade de Odontologia, Universidade Camilo Castelo Branco (UNICASTELO), Campus Fernandópolis- SP Brasil \\ ${ }^{3}$ Cirurgiã-Dentista
}

\section{Resumo}

O Fibroma Ossificante Periférico é uma lesão nodular caracterizada como hiperplásica inflamatória reacional sendo considerada uma das patologias bucais mais comuns, ocorre quase que exclusivamente na gengiva inserida e geralmente envolve a papila interdental. É uma entidade patológica que suscita muitas controvérsias em relação à sua natureza, pois tem etiologia desconhecida. Cálculos dentais, placa bacteriana, próteses mal adaptadas e outros fatores iatrogênicos, alimentos duros durante a mastigação, deglutição e fala, são considerados fatores irritantes gengivais podendo influenciar neste crescimento reacional. Apresenta como aspecto histopatológico mais marcante a presença de calcificações em várias formas e tamanhos. O exame radiográfico é de grande valor para o diagnóstico, visto que focos radiopacos centrais, podem estar associados ou não a discreta reabsorção da crista do rebordo em sua base. Estudos radiológicos, em alguns casos, mostram na massa de tecido mole área de calcificação. O propósito deste trabalho foi relatar um caso clínico de fibroma ossificante periférico, suas características clínicas, radiográficas e histopatológicas, reforçando a importância dos exames complementares no diagnóstico desta patologia. Concluiu-se que o fibroma ossificante periférico é uma lesão reacional da gengiva, nodular e assintomática. Necessita de exame histopatológico para a confirmação do diagnóstico. $\mathrm{O}$ exame radiográfico é de grande importância, pois dependendo da fase de desenvolvimento da lesão esta pode apresentar-se com discretos pontos de calcificações, sem envolvimento do osso subjacente.

Descritores: Paracoccidioidomicose; Mucosa bucal; Manifestações Bucais; Epidemiologia.

\begin{abstract}
Peripheral ossifying fibroma is a nodular lesion characterized as inflammatory and hyperplasic reaction. It is considered one of the most common oral diseases and occurs almost exclusively in the attached gingiva. Usually involves the interdental papillae. It is a pathological entity that raises many controversies regarding their nature, although the unknown etiology. Dental calculus, plaque, illfitting dentures and other iatrogenic factors, food during chewing, swallowing and speaking are considered gingival irritants and may influence this reaction growth. The differential diagnosis is made with the fibrous hyperplasia, peripheral giant cell lesion and pyogenic granuloma. Only clinical findings are not sufficient for the diagnosis of peripheral ossifying fibroma, as both their clinical appearance as developments may be similar to pyogenic granuloma and peripheral granuloma and giant cells. The most striking histologic features is the presence of calcifications. Radiographic examination is useful for the diagnosis, since central radiopaque areas corresponding to mineralization, with or without discrete resorption rim crest in its base, can be identified. Radiologic studies, in some cases, show the mass soft tissue calcification area. This study was to report a case of peripheral ossifying fibroma, their clinical and radiographic features and the histopathological, reinforcing the importance of laboratory tests in the diagnosis of this pathology. We concluded that peripheral ossifying fibroma is a reaction injury gum, nodular and asymptomatic which requires histopathologic examination to confirm the diagnosis. Radiographic examination is of great value because of the injury depending on the development stage this may present with discrete points of calcifications without involvement of the underlying bone.
\end{abstract}

Descriptors: Paracoccidioidomycosis; Mouth Mucosa; Oral Manifestations; Epidemiology.

\section{Resumen}

El fibroma osificante periférico es una lesión nodular caracterizada como reacción inflamatoria hiperplásica se considera una de las enfermedades bucales más comunes se produce casi exclusivamente en la encía adherida y por lo general implica la papila interdental. Es una entidad patológica que genera muchas controversias en cuanto a su naturaleza, aunque la etiología desconocida. El cálculo dental, la placa, las prótesis dentales mal ajustadas y otros factores iatrogénicos, alimentos durante la masticación, deglución y el habla se consideran irritantes gingivales pueden influir en el crecimiento de esta reacción. Presenta como histopatología más llamativa la presencia de calcificaciones (sustancias mineralizadas). El examen radiográfico es útil para el diagnóstico, ya que las áreas centrales opacos a la radiación correspondiente a la mineralización, asociados o no con el borde de absorción discreta de la cresta en la base, que puedan ser identificados. Los estudios radiológicos, en algunos casos, muestran el área masa calcificación de tejidos blandos. Este estudio tuve como objetivo presentar un caso de fibroma osificante periférico, las características clínicas y radiográficas e histopatología, lo que refuerza la importancia de las pruebas complementarias en el diagnóstico de esta patología. Se concluí que el fibroma osificante periférico es una lesión reactiva de la encía, nodular y asintomática. Requiere el examen histopatológico para confirmar el diagnóstico. El examen radiográfico es de gran valor ya que dependiendo de la fase de desarrollo de la lesión puede presentarse con puntos discretos de calcificaciones y sin afectación del hueso subyacente.

Descriptores: Paracoccidioidomicosis; Mucosa Bucal; Manifestaciones Bucales; Epidemiología. 


\section{INTRODUÇÃO}

O fibroma ossificante periférico (FOP) é considerado um patologia bucal bastante comum sendo caracterizado como uma lesão hiperplásica inflamatória reacional. O FOP ocorre quase que exclusivamente na gengiva inserida e geralmente envolve a papila interdental. Cálculos dentais, placa bacteriana, próteses mal adaptadas, restaurações em excesso e alimentos duros durante a mastigação, deglutição e fala, são considerados fatores irritantes gengivais podendo influenciar neste crescimento reacional $^{1,2,3,4}$.

O fibroma ossificante periférico é uma lesão nodular de natureza reacional, tendo como diagnóstico diferencial a hiperplasia fibrosa, lesão periférica de células gigantes e o granuloma piogênico. Embora o FOP pareça ter etiologia desconhecida, alguns autores o consideram como processo inflamatório ou reacional, enquanto outros sugerem processos neoplásicos ${ }^{5,6,7}$.

Esta patologia foi primeira descrita por Menzel, em 1842, mas foi Montgomery (1927), que a denominou de fibroma ossificante periférico. Por estar incluída nas lesões gengivais reacionais, também podem ser chamadas genericamente de "epúlides". Muitos são os termos usados para definir esta estrutura, como: epúlide fibróide ossificante, fibroma periférico com calcificação; granuloma fibroblástico calcificante $^{8,9,10}$.

O FOP é descrito como massa avermelhada ou rosada, em geral séssil, menor que 2 centímetros, podendo raramente assumir dimensões maiores. Autores relatam que o FOP ocorre exclusivamente na gengiva, apresentando-se como massa nodular pedunculada ou séssil, assintomática e de crescimento lento, que usualmente desenvolve-se na papila interdental. A superfície pode estar intacta ou ulcerada. O FOP pode causar o deslocamento dos dentes ${ }^{1,5,8}$.

Esta lesão aparece como um crescimento focal de tecido bem delimitado e seu aspecto histopatológico mais marcante consiste na presença de focos mineralizados em um estroma de tecido conjuntivo com inúmeros fibroblastos. Provavelmente, o produto mineralizado origina-se de células do periósteo ou ligamento periodontal ${ }^{3,8}$.

O exame radiográfico é de valia para o diagnóstico, visto que focos radiopacos centrais correspondendo à mineralização, associados ou não a discreta reabsorção da crista do rebordo em sua base, podem ser identificados. Estudos da peça cirúrgica mostram uma massa de tecido mole, que, em alguns casos apresentam uma área de calcificação. Na grande maioria dos casos não há envolvimento do osso subjacente visível na radiografia. Com a maturação da lesão, o material calcificado aumenta e se coalesce, explicando assim, o aumento da radiopacidade da lesão na radiografia ${ }^{5,8,9}$.

O tratamento consiste na remoção cirúrgica, incluindo o ligamento periodontal e o periósteo com vigorosa raspagem da região de modo a evitar recidiva, o que é frequente em $15 \%$ dos casos ${ }^{1,5}$.

\section{CASO CLÍNICO}

Paciente do gênero feminino, 26 anos, leucoderma compareceu a clínica de Odontologia da Unicastelo com queixa de um crescimento gengival entre os dentes canino e pré-molar inferiores por lingual, dificultando a fala e mastigação. A paciente relatou durante a anamnese que a lesão apresentava crescimento lento, indolor e sem sangramento. No exame clínico intrabucal, verificou-se uma massa nodular na região de gengiva inserida, pediculada, de coloração rósea, com pequenos pontos ulcerados, e de consistência firme, medindo aproximadamente $2,0 \mathrm{~cm}$ de diâmetro. A lesão estava causando deslocamento dos dentes 43 e 44 e prejudicando o repouso da língua no assoalho de boca (Figuras 1 e 2), com a presença de cálculos dentários na região, indicando a péssima higienização bucal da paciente.

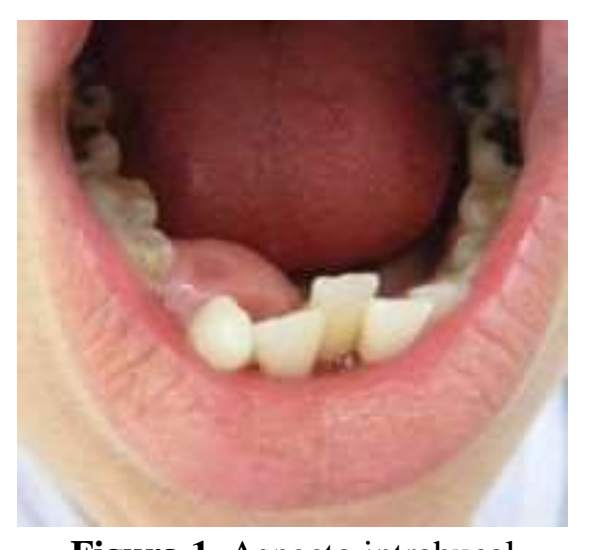

Figura 1. Aspecto intrabucal

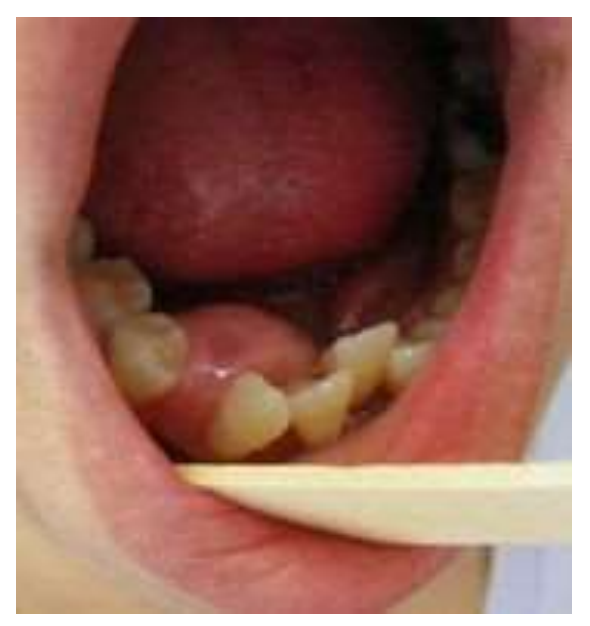

Figura 2. Aspecto da lesão apresentando tumefação e afastamento dos dentes 43 e 44

Realizados os exames radiográficos (panorâmico e periapical da região), observou-se a presença de calcificações no interior da lesão (Figuras 3 a 5). As hipóteses diagnósticas foram de granuloma piogênico, hiperplasia fibrosa inflamatória e fibroma ossificante periférico. Após uma biópsia incisional, o resultado foi fibroma ossificante periférico. Foram realizados orientação de escovação e motivação da 
paciente quanto aos métodos de higiene bucal, raspagem e alisamento radicular a fim de melhorar as condições de saúde bucal.

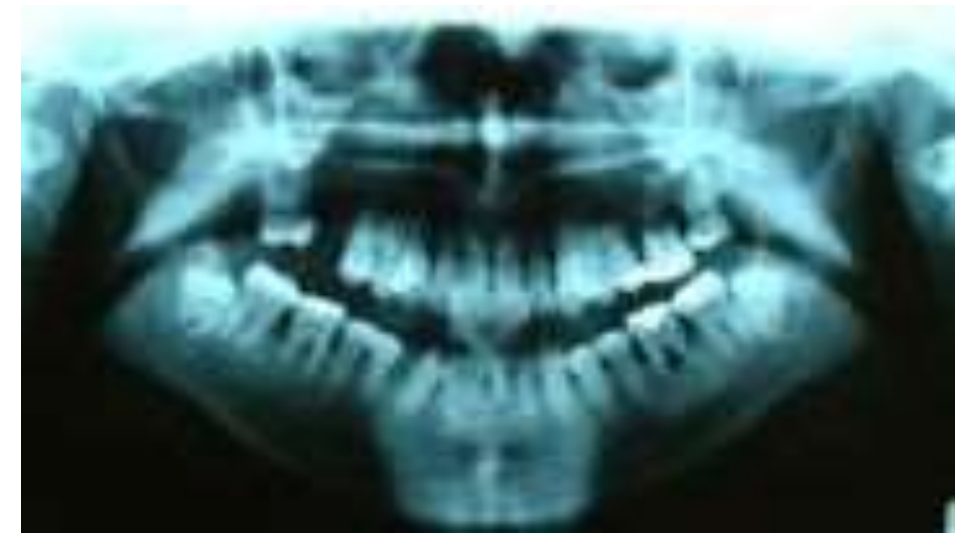

Figura 3. Aspectos radiográficos - radiografia panorâmica

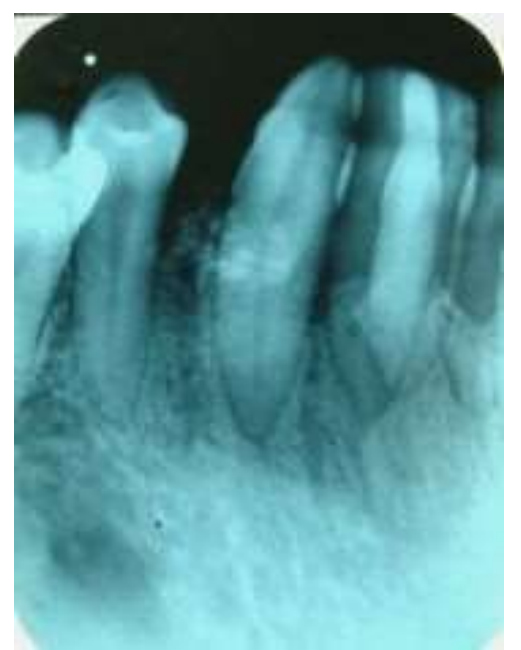

Figura 4. Aspecto radiográfico - radiografia periapical. Focos radiopacos

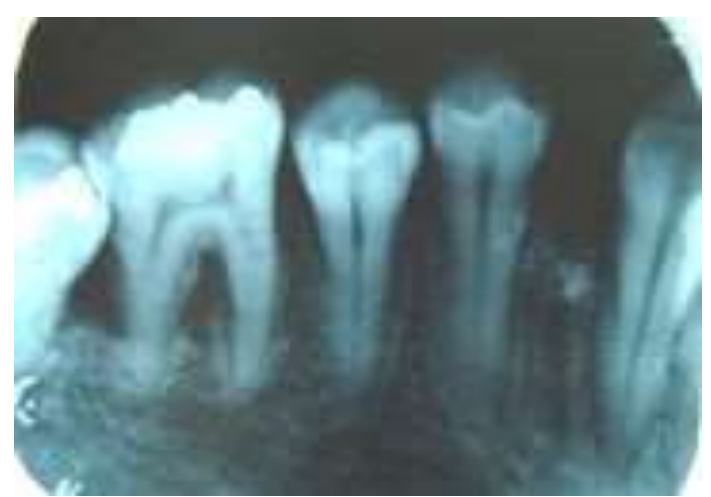

Figura 5. Aspecto radiográfico - radiografia periapical. Focos radiopacos

A remoção cirúrgica foi total da lesão, com intensa curetagem e raspagem subgengival dos dentes adjacentes por vestibular e palatino (Figuras 6 e 7), sendo a peça enviada para o exame histopatológico.

Os cortes histológicos revelaram fragmento de mucosa bucal caracterizado pela proliferação de células fusiformes, de citoplasma amplo e claro. O estroma estava constituído por tecido conjuntivo denso com feixes de fibras colágenas dispostas paralelamente entre si, exibindo discreto infiltrado inflamatório predominantemente mononuclear localizado de forma irregular em todo o espécime, vasos sanguíneos proliferados de diversos tamanhos. Observou-se ainda, fragmento esférico de tecido mineralizado completando os cortes examinados (Figura 8).

O pós-operatório constitui-se de duas avaliações com intervalos de sete dias, evidenciando completa regeneração tecidual (Figura 9).

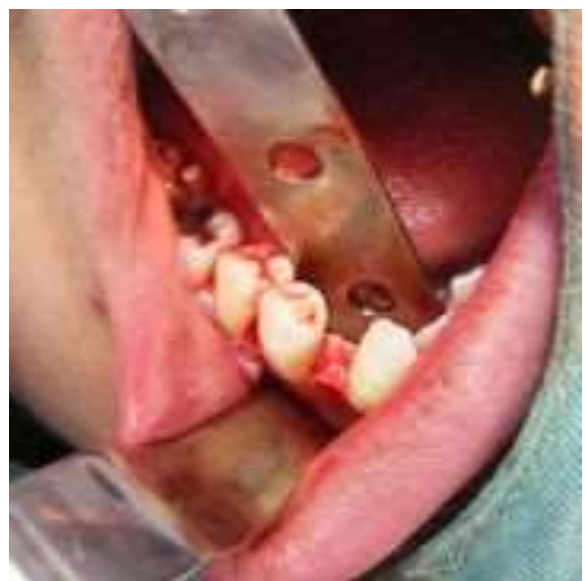

Figura 6. Aspecto transoperatório

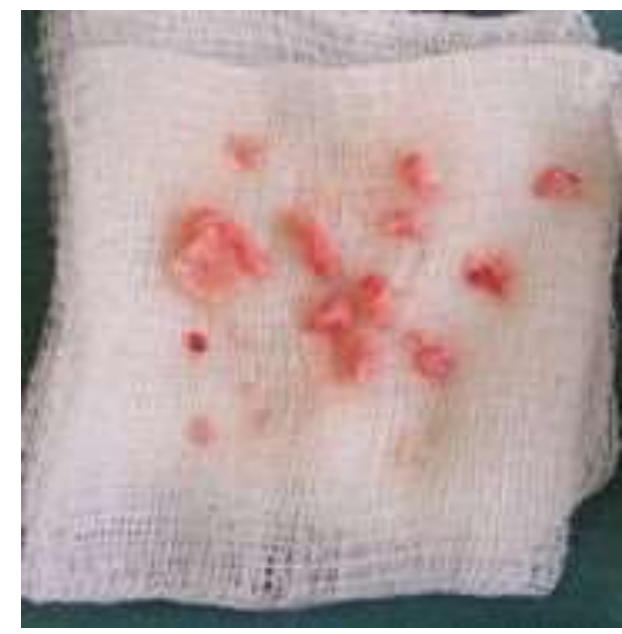

Figura 7. Fragmentos da lesão

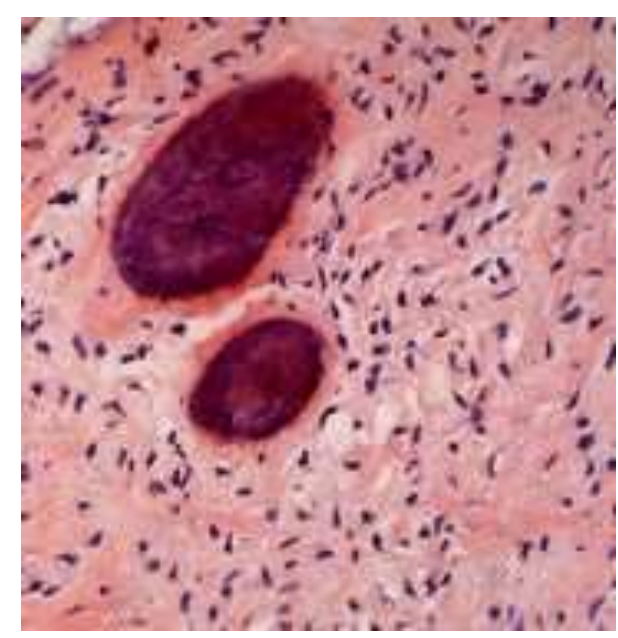

Figura 8. Aspecto histopatológico exibindo tecido mineralizado

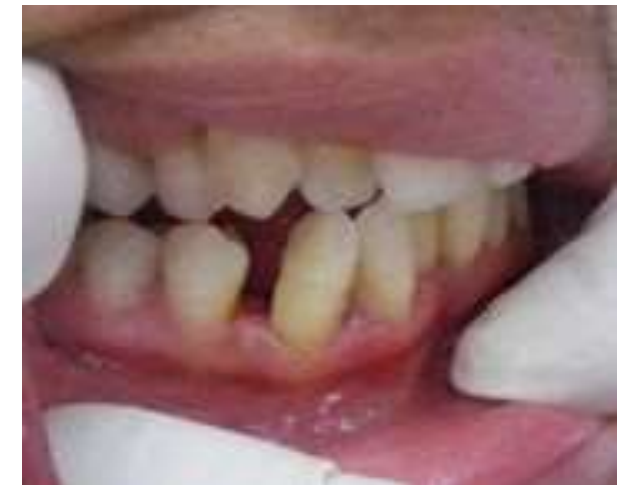

Figura 9. Pós-operatório após 14 dias evidenciando completa regeneração tecidual

\section{DISCUSSÃO}

O fibroma ossificante periférico é uma entidade patológica que suscita muitas controvérsias em relação à sua natureza ${ }^{10}$. Para Kumar et al. ${ }^{6}$ (2006), o FOP é uma lesão de crescimento gengival comum, única parecendo relacionar-se ao ligamento periodontal. Freitas et al. ${ }^{10}(2004)$, apresentaram o FOP como um crescimento não neoplásico da gengiva classificado como uma lesão hiperplásica inflamatória reativa, tendo origem incerta mas parecendo estar associado ao ligamento periodontal. Marcucci et al. 
${ }^{5}$ (2005) descreveram o FOP como lesão de natureza reacional, localizada exclusivamente na gengiva inserida e papila interdental. Apesar de apresentar uma etiopatogenia incerta, existem bons motivos para considerar sua origem das células do ligamento periodontal: ocorrência exclusiva na gengiva (papila interdental), a proximidade da gengiva com 0 ligamento periodontal, a presença das fibras oxitalânicas no interior da matriz mineralizada em algumas lesões e a resposta fibrocelular no FOP semelhante a outras lesões gengivais reativas com origem no ligamento periodontal $^{6,7}$.

Mesquita et al. ${ }^{4}(1998)$, realizaram um estudo, a fim de esclarecer a discussão em torno da etiologia dos fibromas ossificantes, concluindo que o FOP é uma lesão reativa da gengiva e o FO, uma lesão neoplásica. Freitas et al. ${ }^{10}(2004)$, reforçaram que FOP e FO são entidades patológicas com características histológicas semelhantes, ambas se originam no ligamento periodontal, sendo que o diagnóstico diferencial se centraliza nos achados clínicos, o FO é uma lesão que acomete frequentemente a região posterior da mandíbula, podendo provocar expansão óssea da região afetada e atingir grandes dimensões.

Traumas ou irritantes locais podem estar relacionados à lesão, como restos radiculares, placa e cálculos dentais, fatores iatrogênicos (como restaurações mal adaptadas, com excessos) e traumas mastigatórios $^{1,10}$. No caso clínico apresentado a lesão estava localizada na porção lingual da gengiva inserida na mandíbula, local susceptível a traumas mastigatórios, sendo única e com presença abundante de cálculo gengival na região (talvez sugerindo uma relação com a etiologia da lesão).

Segundo Neville et al. ${ }^{8}(2004)$, alguns FOP, devido as suas características clínicas e histopatológicas semelhantes, são considerados granulomas piogênicos que na fase inicial sofrem maturação fibrosa e subsequente calcificação, no entanto nem todos os FOP se desenvolvem dessa maneira. Lourenço et al. ${ }^{3}(2004)$, apresentaram um caso clínico em que a lesão inicial era um granuloma, e sua recidiva já se tratava de um FOP, reforçando a possibilidade de um FOP ser a maturação de um granuloma piogênico. Kumar et al. ${ }^{6}(2006)$, reforçaram que somente achados clínicos não são suficientes para o diagnóstico de FOP, pois tanto a sua aparência clínica como a evolução podem ser parecidos ao granuloma piogênico e ao granuloma periférico de células gigantes. O FOP quando em estágio inicial, pode ser clinicamente diagnosticado erroneamente como granuloma piogênico ${ }^{1}$.

O granuloma piogênico, é uma lesão nodular que se diferencia do FOP através dos achados histopatológicos que evidenciam hiperplasia inflamatória com presença de neutrófilos e neoformação vascular. Em relação ao granuloma periférico de células gigantes, sua localização não envolve só a gengiva, mas também a mucosa alveolar de desdentados. Tem evolução rápida e associada a traumas (próteses sobre espículas ósseas ou traumas decorrentes de exodontias), radiograficamente revela reabsorção em forma de taça do osso subjacente, e encontra-se hemorragia abundante em toda massa celular $^{8,11}$.

$\mathrm{Na}$ literatura são encontrados poucos estudos relacionados ao FOP, o que torna as informações referentes às características destas lesões pouco conclusivas $^{10}$. Considerando os aspectos clínicos desta entidade, Neville et al. ${ }^{8}$ (2004) mencionaram que esta se apresenta como uma massa gengival firme, frequentemente séssil, de crescimento lento e recoberta por uma mucosa aparentemente normal. Marcucci et al. ${ }^{5}$ (2005), a descreveram como massa avermelhada, geralmente séssil, não superior a dois centímetros. Tomassi ${ }^{11}(1998)$ refere-se a esta lesão como sendo pediculada e eventualmente séssil. Em um estudo com 67 casos clínicos, Freitas et al. ${ }^{10}$ (2006) constataram que a implantação pediculada predominou, com lesões tendendo ao crescimento lento, de coloração rósea e de consistência fibrosa. Geralmente a lesão é assintomática até o crescimento produzir tumefação visível e deformidade moderada; o deslocamento dos dentes pode ser uma de suas características clínicas. Por ter um crescimento lento, as corticais ósseas e a mucosa subjacente ficam usualmente intactas ${ }^{9}$. A lesão relatada no caso clínico apresentava características semelhantes sendo uma massa focal de tecido rósea, bem delimitada, com pequenas ulcerações, pediculada e de crescimento lento, fibrosa, medindo $2 \mathrm{~cm}$ e causando o afastamento dos dentes vizinhos.

A maior ocorrência desta patologia está no sexo feminino ${ }^{4,12,13}$, sendo que Neville et al. ${ }^{8}(2004)$ afirmaram que a tumefação pode ocorrer em qualquer idade. A localização mais frequente dessa lesão, segundo alguns autores é a região anterior da maxila ${ }^{1,4}$. Neville et al. ${ }^{8}(2004)$ citaram a região vestibular da mandíbula como maior ocorrência, e Freitas et al. ${ }^{10}(2004)$ relataram um caso clínico de FOP na mandíbula coincidindo com a região anatômica do caso relatado.

Quanto ao aspecto radiográfico, Poon et al. ${ }^{1}$ (1995) citaram, que este pode ser um exame auxiliar de grande importância, porém somente quando a lesão for madura, mesmo assim existe a possibilidade de não se verificar nenhuma alteração na imagem radiográfica. Pequenos ajustes na exposição podem ser feitos para que pequeninos focos radiopacos sejam identificados. Kumar et al. ${ }^{6}(2006)$ afirmaram que a lesão pode apresentar vários estágios de fibroma com ossificação significando FOP, contudo, calcificações 
ou ossificações podem não ser evidentes em todos os casos, particularmente em estágios primários de crescimento da lesão, sendo que achados radiográficos nem sempre são visíveis nos FOP. A lesão apresenta aspecto radiográfico variável, dependendo da fase de desenvolvimento ${ }^{9,14}$. No caso clínico a lesão mostrou-se na imagem radiográfica como uma massa de tecido mole com discretos pontos de calcificações, sem envolvimento do osso subjacente.

Diferentes tipos de calcificações podem ser encontrados nos cortes microscópios, como; osso lamelar maduro, osso imaturo, focos de calcificações distróficas e um material amorfo, que alguns autores classificam ser semelhante ao cemento ${ }^{1}$. O diagnóstico definitivo de FOP deve ser feito através da avaliação histopatológica das biópsias, onde usualmente são observadas as seguintes características microscópicas: 1) tecido fibroso benigno variando a quantidade de fibroblastos e a deposição de colágeno; 2) proliferação endotelial esparsa e abundante; 3) material mineralizado maduro, lamelar ou tecido osteóide, material amorfo- semelhante ao cemento, ou calcificações distróficas ${ }^{6,15}$.

O tratamento do FOP preconizado é a excisão cirúrgica completa da lesão, devendo ser suficientemente profunda para incluir o periósteo e o ligamento periodontal ${ }^{1,810}$. Embora, usualmente a excisão seja curativa, tem sido relatada uma taxa de $16 \%$ de recorrências ${ }^{1,8,16}$. A identificação e remoção de possíveis agentes irritantes é de grande importância para o tratamento ${ }^{4,17}$. O acompanhamento dos pacientes é indispensável para evitar recorrências da lesão sendo importante enfatizar a necessidade de manutenção da higiene bucal para prevenção desta e outras lesões ${ }^{3}$.

\section{CONCLUSÃO}

Diante do caso apresentado podemos concluir que o Fibroma Ossificante Periférico necessita de exame histopatológico para a confirmação do diagnóstico e que o exame radiográfico é de grande valia, pois dependendo da fase de desenvolvimento da lesão esta pode apresentar-se com discretos pontos de calcificações sem envolvimento do osso subjacente.

\section{REFERÊNCIAS}

1. Poon CK, Kwan PC, Chao SY. Giant peripheral ossifying fibroma of the maxilla: report of a case. J Oral Maxillofac Surg. 1995;53(6):695-8.

2. Freitas TMC, Soares AF, Freitas RA, Galvão HC, Souza EL. Fibroma ossificante periférico: estudo de 67 casos em Natal, RN. Rev ABO Nac. 2006;14(2):113-6.

3. Lourenço SQC, Balassiano KZ, Nunes AJG. Fibroma ossificante periférico: relato de caso clínico e histórias de recidivas. Rev Bras Odontol. 2004;61(2):127-9.

4. Mesquita RA, Sousa SCOM, Araújo NS. Proliferative activity in peripheral ossifying fibroma and ossifying fibroma. J Oral Pathol Med. 1998;27(2):64-7.

5. Marcucci G. Fundamentos de OdontologiaEstomatologia. Rio de Janeiro: Guanabara Koogan; 2005.

6. Kumar SKS, Ram S, Jorgensen MG, Charles FS, Parish PS. Multicentric peripheral ossifying fibroma. J Oral Sci. 2006;48(4):239-43.

7. Yadav R, Gulati A. Peripheral ossifying fibroma: a case report. J Oral Sci. 2009;51(1):151-4.

8. Neville BW, Damm DD, Allen CM, Bouquot JE. Patologia Oral \& Maxilofacial. 2 ed. Rio de Janeiro: Guanabara Koogan; 2004.

9. Ferraz TM, Nogueira TO. Histoquímica dos tecidos mineralizados nas lesões de displasia fibrosa e fibroma cemento-ossificante periférico. Rev Odontol UNESP. 1998;27(1):87-98.

10. Freitas TMC, Farias JG, Freitas VZ, Ramos MESP, Alves MF, Ramos Jr RP. Fibroma ossificante periférico: relato de um caso clínico. Rev ABO Nac. 2006;12(5):287-291.

11. Tomassi AF. Diagnóstico em Patologia Bucal. 2 ed. Curitiba: Pancast; 1989.

12. Daley TD, Wysocki GP. Peipheral odontogenic fibroma. Oral Surg Oral Med Oral Pathol. 1994;78(3):329-35.

13. Moon WJ, Choi SY, Chung EC, Kwon KH, Chae SW. Peripheral ossifying fibroma in the oral cavity: CT and MR findings. Dentomaxillofac Radiol. 2007;36(3):180-6.

14. Liu Y, Wang H, You M, Yang Z, Miao J, Shimizutani K et al. Ossifying fibromas of the jaw bone: 20 cases. Dentomaxillofac Radiol. 2010;39(1):57-63.

15. Sanchis JM, Peñarrocha M, Balaguer JM, Camacho F. Fibroma cemento-osificante mandibular: Presentación de dos casos y revisión de la literatura. Med Oral. 2004;9(1):69-73.

16. Galdeano-Arenas M, Crespo-Pinilla JI, ÁlvarezOtero R, EspesoFerrero A, Verrier-Hernández A. Fibroma cemento-osificante gingival mandibular. presentación de un caso. Med Oral. 2004;9(2):1769.

17. Ong $\mathrm{AH}$, Siar $\mathrm{CH}$. Cemento ossifying fibroma with mandibular fracture. Case report in a young patient. Aust Dent J. 1998;43(4) :229-33. 
Arch Health Invest (2016) 5(2): 106-111

\section{CONFLITO DE INTERESSES}

Os autores declaram não haver conflitos de interesse.

\section{AUTOR PARA CORRESPONDÊNCIA}

Lucieni Cristina Trovati Moreti

lucienimoreti@hotmail.com

Submetido em 22/03/2016

Aceito em 05/04/2016 